\section{Influence of a Variety of Grain Boundary Structures on Grain Boundary Segregation}

There are some available models to describe the behavior of the grain boundary segregation in polycrystalline materials. The equilibrium segregation theory proposed by McLean ${ }^{1)}$ describes thermal equilibrium of elements in a matrix and grain boundaries. He derived a fundamental equation of relationship between a segregated amount and a bulk concentration with thermodynamical parameters of the free encrgy change for the segregation as follows.

$$
X_{b} /\left(1-X_{b}\right)=X_{c} /\left(1-X_{c}\right) \exp (\Delta G / k T)
$$

where, $\Delta G$ : the free energy change for the segregation

$k$ : the Boltzmann constant

7: an equilibrium temperature

$X_{b}, X_{c}$ : a fraction occupied by segregants in the segregation site and the concentration in bulk, respectively.

Guttmann expanded the model to a multi-component system with the regular solution model, and emphasized an interaction between impurities and alloying elements in a ternary system. ${ }^{2}$ Hondros and Seah took into account the effect of a repulsive interaction of impurities at a high segregation level using a thermodynamical model. ${ }^{3 \text { ) }}$

The above models on the equilibrium segregation assumed that the thermodynamical parameters are constant, and did not take account of a variety of type of grain boundaries. Contrary to the assumption, however, atomistic investigations on the grain boundary structure have shown that grain boundaries with various types of structure can become segregation sites. ${ }^{4,5)}$ Ashby et al., for instance, described the segregation site with a polyhedral model of grain boundaries, ${ }^{4)}$ and it suggested dependence of the grain boundary structure on the segregated amount. Briant considered that the effect should be attributed to a variation of the segregation with fractured boundary planes. ${ }^{6)}$ Although White and Coghlan investigated a spectrum of binding cnergy between impurities and grain boundaries using a dislocation model of grain boundaries, ${ }^{7}$ ) it has not been applied to a general case. Thus, the cffect of a variation of the segregation with grain boundary structures in polycrystalline materials should also be discussed in the application of the equilibrium segregation model to an analysis of the results.

The grain boundary segregation can generally be examined by analyzing in intergranular fractured surfaces in an ultra high vacuum by means of microprobe scanning Auger electron microscopy. The analysis reveals that the segregated amount depends on the fractured plane even in a sample. There may be two reasons for the variation of the segregation with the fractured grain boundary plane. One is the variation of the density of the segregation site or the degree of the saturated segregation for impurity atoms. If the segregated amount is evaluated with the average value of the results over the grain boundaries, the effect of the density is eliminated. The other is the variation of the energy of the segregation on each boundary plane due to all kind of grain boundary structures. The effect also results in the variation of the segregation with the boundary plane at a given temperature even in the same density of the segregation site. The high energy for the segregation or the deep trapping of impurities to grain boundaries causes a highly concentrated segregation at a temperature. Therefore, an average segregated amount which is obtained experimentally must involve the effect of the distribution of the segregation energy change due to the structure. The average amount, $X_{b}^{\mathrm{av}}$, is then given by

$$
X_{b}^{\mathrm{av}}=\int_{0}^{\infty} X_{b}\left(\Delta G_{i}\right) f\left(\Delta G_{i}\right) d \Delta G_{i}
$$

where, $f\left(\Delta G_{i}\right)$ : a distribution function of the energy change for the segregation

$X_{b}\left(\Delta G_{i}\right)$ : given by $\mathrm{Eq}$. (1) with a segregation energy change $\Delta G_{i}$.

The entropy term does not contribute to temperature dependence of the segregated amount, so that it should be discussed how the apparent enthalpy from results observed from $\mathrm{Eq}$. (2) deviates from the simple McLean's relation. The distribution of the energy may be described with a mean enthalpy and a deviation of it, because the segregation behavior and the grain boundary energy depend on the grain boundary structure. ${ }^{5,6)}$ Here, for simplicity a Gaussian distribution was taken, since the fundamental feature of the effect of the distribution discussed later is almost independent of the shape as far as the segregation energy has a broad distribution.

The segregation enthalpy of $50 \mathrm{~kJ} / \mathrm{mol}$ is a typical value for the grain boundary segregation, which is estimated in phosphorus in iron around $1100 \mathrm{~K}$ approximately from the concentration dependence of the segregation. ${ }^{8}$ If the segregation of the mean segregation enthalpy is accompanied with a standard deviation from it, the effect may be evaluated. Fig. 1 shows temperature dependence of segregation calculated with the simple McLean's relation and the relation taking into account the effect of the distribution of the segregation enthalpy in the exponential form. The calculation was carried out under conditions that the mean segregation enthalpy is $50 \mathrm{~kJ} / \mathrm{mol}$ and the standard deviation of the segregation is $30 \%$ 


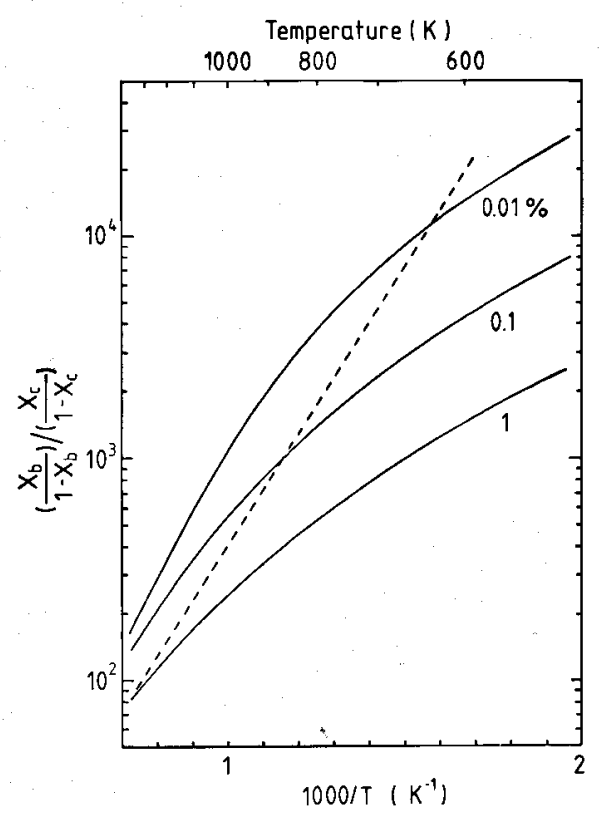

The numbers in the figure: The bulk concentration of the solutes (at \%)

Fig. 1. Temperature dependence of the simple McLean's relation (dashed lines) and the relation taking into account the enthalpy distribution (solid lines).

of the mean value, $15 \mathrm{~kJ} / \mathrm{mol}$. If the distribution of the segregation enthalpy is decreased, the plot with the distribution approaches to the result of the simple McLean's relation. The deviation of the plot with energy distribution from the simple McLean's relation results from the distribution of the segregated elements to a variety of grain boundaries; in a variety of grain boundaries, grain boundaries with high segregation energy reveal higher segregation than that predicted by the simple McLean's model at elevated temperatures, and the low segregation at boundaries with a low energy is observed at low temperatures. In other words, the population of the segregation strongly affects the observable segregated amount.

Fig. 2 shows temperature dependence of the observable cnthalpy and the entropy obtained from the plots with the deviation of the segregation enthalpy in Fig. 1. The observable enthalpies are smaller than the average value of $50 \mathrm{~kJ} / \mathrm{mol}$ over practical temperatures, depending on the concentration. This generally indicates that the apparent segregation enthalpy is lower than the mean value of the segregation enthalpy, and the apparent entropy becomes larger due to the effect of the distribution of the segregation enthalpy, although the effect is dependent on the distribution shape. Erhart and Grabke studied the equilibrium grain boundary segregation of phosphorus in iron, and obtained the enthalpy of about $34 \mathrm{~kJ} /$ mol. ${ }^{9)}$ Since the measurement may involve the effect of a variety of segregation sites, the actual mean segregation enthalpy may be larger than the measured. The present discussion suggests that it is difficult to estimate a strict thermodynamical parameters for the segregation because of the various kind of the segregation site.

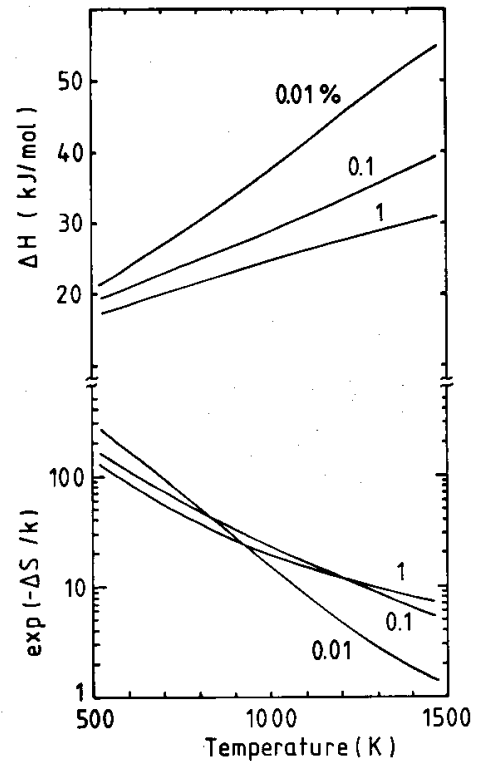

Fig. 2. Temperature dependence of the segregation enthalpy and entropy obtained from solid lines in Fig. 1 .

It should be noted that there is another difficulty in discussing the grain boundary segregation strictly, which is the evaluation of an exact concentration of the segregated amount at grain boundaries. Because it is difficult to estimate the exact volume or the exact thickness of a segregated layer which is necessary to obtain the segregated concentration, for complexity of atomistic structures on grain boundaries.

Fig. 1, however, indicates a method to estimate more real mean thermodynamical parameters. A half amount of the saturated segregation is approximately equal to the value expected in the simple McLean's theory, and it is shown by intersections of the lines of the simple McLean line and the lines with the distribution of the segregation enthalpy in Fig. 1. This means that a half value of the saturated segregation obtained in samples with various compositions can give the mean value of the segregation enthalpy and entropy.

It is a concluding remark that the effect of the distribution of the segregation energy due to all kinds of segregation sites can result in a deviation of the real thermodynamic parameters, generally underestimation of the segregation enthalpy. This is to be noted in a quantitative thermodynamical analysis for experimental results on the grain boundary segregation.

\section{REFERENCES}

1) D. McLean: Grain Boundaries in Metals, Oxford Univ. Press, London, (1957), 116.

2) M. Guttmann: Surface Sci., 53 (1975), 213.

3) E. D. Hondros and M. P. Seah: Metall. Trans, A, 8A (1977), 1363.

4) M. F. Ashby, F. Spaepen and S. Williams: Acta metall., 26 (1978), 1647.

5) A. P. Sutton and V. Vitek: Acta metall., 30 (1982), 2011.

6) C. L. Briant: Acta metall., 31 (1983), 257.

7) C. L. White and W. A. Coghlan: Metall. Trans. A, 8A 
ISIJ International, Vol. 30 (1990), No. 11

(1977), 1403

8) S. Suzuki, M. Obata, K. Abiko and H. Kimura: Trans. Iron Steel Inst. Jpn., 25 (1985), 62.

9) H. Erhart and H. J. Grabke: Metal Sci., 15 (1981), 401.

(Received on May 22, 1990; accepted in the final form on July 20,1990)

\section{Shigeru SUZUKI}

Materials Gharacterization Research Lab., Nippon Steel Corporation,

Ida, Nakahara-ku, Kawasaki, Kanagawa-ken, 211 Japan. 